\title{
EchoGéo
}

\section{Politiques et pratiques de la résilience}

Introduction

\section{Antoine Le Blanc et Thierry Nicolas}

\section{(2) OpenEdition}

Journals

Édition électronique

URL : https://journals.openedition.org/echogeo/13451

DOI : 10.4000/echogeo.13451

ISSN : 1963-1197

Éditeur

Pôle de recherche pour l'organisation et la diffusion de l'information géographique (CNRS UMR 8586)

Référence électronique

Antoine Le Blanc et Thierry Nicolas, "Politiques et pratiques de la résilience », EchoGéo [En ligne], 24 I 2013, mis en ligne le 10 juillet 2013, consulté le 11 août 2021. URL : http://journals.openedition.org/ echogeo/13451; DOI : https://doi.org/10.4000/echogeo.13451

Ce document a été généré automatiquement le 11 août 2021.

EchoGéo est mis à disposition selon les termes de la licence Creative Commons Attribution - Pas d'Utilisation Commerciale - Pas de Modification 4.0 International (CC BY-NC-ND) 


\title{
Politiques et pratiques de la résilience
}

\author{
Introduction
}

Antoine Le Blanc et Thierry Nicolas

1 Ce dossier porte sur un concept qui suscite un intérêt croissant en géographie depuis quelques années : la résilience. Le concept n'est pas à proprement parler nouveau car de nombreuses disciplines y ont recours à l'image de l'écologie (Holling l'utilise dès 1973 pour décrire des systèmes évolutifs, qui absorbent les chocs, par opposition à des systèmes stables), des sciences physiques (où il désigne l'élasticité, la plasticité des matériaux soumis à des chocs), ou encore de la psychologie (qui l'a popularisé en France, notamment, suite aux travaux de B. Cyrulnik).

2 L'utilisation du concept de résilience en sciences géographiques semble répondre aux bouleversements socio-économiques et technologiques mondiaux extrêmement rapides des dernières décennies qui ont modifié la perception et les modalités de gestion des risques, dans les sociétés industrialisées tout au moins (Beck, 2001). C'est notamment à la suite de la Décennie Internationale des Risques et de la Stratégie Internationale de Réduction des Catastrophes initiée dans les années 1990, puis d'un colloque au MIT intitulé "The resilient city: trauma, recovery and remembrance ", que la notion est intronisée comme un paradigme nouveau en géographie (Vale et Campanella, 2005). Alors que le modèle théorique et opérationnel dominant de la prévention a montré certaines limites, la résilience semble ouvrir de nouvelles perspectives en étant perçue comme «la capacité d'un système social (par exemple une organisation, une ville, ou une société) à s'adapter de manière proactive et à se remettre de perturbations perçues, au sein du système, comme non ordinaires et non attendues » (Comfort et al., 2010). La résilience consisterait ainsi à s'éloigner d'un modèle de gestion techniciste, à proposer une gestion qui s'adapte en permanence aux évolutions complexes d'un système territorial, enfin à donner plus de place à des acteurs plus variés et aux facteurs plus sociétaux des risques.

3 Toutefois les contours précis de la notion et surtout sa mise en application sont encore très controversés. Certains critiquent un concept ne pouvant être validé qu'a posteriori, 
ou rattaché à une idéologie spécifique ; d'autres soulignent l'impossibilité de mettre en œuvre des politiques fondées sur un socle aussi mal défini auquel se rattache un entrelacs de notions (résistance, récupération, adaptation, adaptabilité ...) de manière relativement confuse. L'articulation résilience/vulnérabilité est elle-même problématique, au point de constituer un élément récurrent de ce dossier.

4 La plupart des auteurs s'attardent en effet sur ce couple vulnérabilité/résilience. Béatrice Quenault rappelle que la relation entre les deux concepts s'étend de l'opposition à l'inclusion en passant par le chevauchement. Cette étendue des gammes d'approches s'explique entre autres par la volonté de mettre en avant soit une conception statique de la résilience soit, au contraire, une conception dynamique. Pour Bruno Barroca, Maryline Di Nardo et Irène Mboumoua, la résilience, notamment en contexte urbain, semble succéder à la vulnérabilité en raison notamment du passage dans les cindyniques d'une approche analytique à une approche systémique. A la différence de la vulnérabilité, étudier la résilience en ville suppose d'aller au-delà d'une simple recherche de causalité et d'envisager les organisations et les interactions qui donnent corps aux objets urbains. Le succès de la notion serait alors le corollaire des évolutions non seulement de la recherche mais aussi des pratiques d'aménagement. De son côté, Magali Reghezza-Zitt propose de lire la résilience (et la vulnérabilité) à travers le prisme de deux traditions qui se sont construites parallèlement: les risk et hazard researches. Elle souligne la nécessité de prendre en compte les échelles temporelles dans la définition même du concept : au moment où survient la crise, on appellera résilience des actions de résistance ou d'absorption du choc, tandis qu'après la crise et à différentes échelles de temps, la résilience deviendra de plus en plus capacité d'adaptation et de renouvellement.

5 Toutefois, au-delà des controverses théoriques, l'objectif de ce dossier est surtout d'interroger les pratiques que le concept de résilience peut engendrer, sur des territoires variés. En effet, la résilience commence à apparaître (et à se répandre) dans les documents de planification à différentes échelles. La résilience, en tant que modalité de gestion des risques, semble constituer désormais un objectif incontournable des politiques d'aménagement des territoires.

Or, ce dossier montre que les premières applications du concept tel qu'il est mobilisé dans la planification urbaine en France ou ailleurs, loin de rompre avec une gestion du risque techniciste, semble plutôt reprendre d'anciens instruments, conforter les dynamiques visant à gérer le risque par la mise en œuvre de solutions techniques, et s'appuyer encore sur des solutions centrées sur l'aléa. Béatrice Quenault souligne ainsi que la résilience impliquerait de mieux prendre en compte les facteurs plus sociétaux des risques tels que la progression de l'isolement, des inégalités et des fragmentations socio-spatiales, ainsi que les représentations des risques. Mathilde Gralepois, Julie Daluzeau et Clément Oger aboutissent à la même conclusion avec l'étude du PPRI du Val de Tours, et proposent une interprétation plus radicale : par définition, la résilience serait très difficile à appliquer en France, car elle repose sur des paradigmes de collectivité et d'auto-organisation spécifiques à la culture anglo-saxonne.

7 D'autre part, ce dossier souligne également le paradoxe, maintes fois constaté, d'une gestion nécessairement locale des risques, adaptée aux aspérités des territoires et des sociétés, et d'une réalité législative et gestionnaire peu encline à déléguer ce pouvoir de gestion à des acteurs considérés comme non légitimes ou non compétents. Plus radicalement, si la résilience implique que les acteurs politico-administratifs 
traditionnels de la gestion des risques se délestent de pans entiers de cette prérogative au profit d'acteurs plus variés et d'actions couvrant un spectre extrêmement vaste, que reste-t-il de cette « gestion des risques »?

8 Le dernier texte du dossier, rédigé par un collectif de neuf auteurs, propose alors de prendre en compte la notion de "viabilité d'un système " plutôt que celle de résilience. En développant un cas d'étude très spécifique au Guyana, les auteurs montrent que les approches bottom up et participatives peuvent proposer une lecture plus souple et pertinente d'un système territorial, des risques auxquels ce système est confronté (du moins des risques perçus par les sociétés considérées), et des réponses qu'il est possible d'apporter en conséquence. L'angle d'approche est un peu décalé : il s'agit non pas d'identifier d'abord l'aléa, mais les valeurs majeures données à un territoire, qui définissent ensuite une hiérarchisation des risques et donc des crises et de leur gestion, l'objectif étant d'assurer la continuité du système.

\section{BIBLIOGRAPHIE}

Beck U., 2001. La société du risque. Sur la voie d'une autre modernité. Paris, Aubier, Alto.

Cyrulnik B., Duval P. (dir.), 2006. Psychanalyse et résilience. Paris, Odile Jacob.

Comfort L. K., Boin A., Demchak C., 2010. Designing Resilience. Preparing for Extreme Events. Pittsburgh, University of Pittsburgh Press.

Dauphiné A., Provitolo D., 2007. La résilience: un concept pour la gestion des risques. Annales de Géographie, n 654, p. 115-125.

Holling C. S., 1973. Resilience and stability of ecological systems. Annual Review of Ecology and Systematics, $\mathrm{n}^{\circ} 4$, p. 1-23.

Vale J. V., Campanella T. J. (dir.), 2005. The Resilient City. How modern cities recover from disaster. New York, Oxford University Press.

Reghezza-Zitt M., Rufat S., Djament-Tran G., Le Blanc A., Lhomme S., 2012; What Resilience Is Not: Uses and Abuses. Cybergéo, 621, http://cybergeo.revues.org/25554

\section{INDEX}

Thèmes : Sur le Champ - Sur le Terrain

\section{AUTEURS}

\section{ANTOINE LE BLANC}

Antoine Le Blanc, Antoine.Le-Blanc@univ-littoral.fr, est Maître de conférences en géographie à l'Université du Littoral Côté d'Opale, TVES (EA 4477),PRES Lille Nord de France. Il a publié 
récemment :

- Reghezza-zitt M., Rufat s., Djament-Tran G., Le Blanc A., Lhomme s., 2012. What Resilience Is Not: Uses and Abuses. Cybergéo, 621, http://cybergeo.revues.org/25554

- Le Blanc A., 2010. La conservation des ruines traumatiques : un marqueur ambigu de l'histoire urbaine. L'Espace Géographique, $\mathrm{n}^{\circ}$ 3, p. 253-266.

- Zwarterook I., Le Blanc A., 2010. Les risques et pollutions industriels sur le territoire dunkerquois : des perceptions à la concertation. Numéro 2010-07 des Cahiers de la Sécurité Industrielle, 152, Institut pour une Culture de Sécurité Industrielle, Toulouse, 124 p. http://www.icsi-eu.org/francais/ dev_cs/cahiers/

\section{THIERRY NICOLAS}

Thierry Nicolas, thierrynicolas@wanadoo.fr, est Chargé de recherche contractuel à l'Université des Antilles et de la Guyane / UMR Espace-DEV (UMR 228), Centre IRD de Guyane. Il a publié récemment :

- Nicolas Th., 2013.L'aéroport de Saba, le porte-avions des Petites Antilles. Mappemonde, Lieux, $\mathrm{n}^{\circ} 109$, http://mappemonde.mgm.fr/

- Nicolas Th., 2011. Insularité et développement dans les Petites Antilles. In Hartog Th. (dir.), La Caraibe : un espace pluriel en questions, Paris, Karthala, Collection « Terres d'Amérique ». - Nicolas Th., 2010. Nissonologie et tourisme : les diversités insulaires au prisme de l'activité touristique. In Jauze J-M., Combeau Y. (dir.), Géographes et Historiens dans la compréhension et la gouvernance des espaces et sociétés de l'océan Indien, Université de La Réunion, Saint-Denis, «Terres et sociétés indocéaniques». 